\title{
Aspectos Biológicos de Catolaccus grandis (Burks) (Hymenoptera: Pteromalidae), Parasitóide do Bicudo do Algodoeiro
}

\author{
Lúcia H. A. Araujoe Francisco R. Azevedo ${ }^{2}$ \\ ${ }^{1}$ Embrapa Algodão, Caixa postal 174, 58107-720, Campina Grande, PB. \\ ${ }^{2}$ Universidade Federal Rural de Pernambuco (UFRPE), Departamento de \\ Agronomia, 52071, Recife, PE.
}

An. Soc. Entomol. Brasil 26(3): 503-506 (1997)

Biological Aspects of Catolaccus grandis (Burks) (Hymenoptera: Pteromalidae) Parasitoid of the Cotton Boll Weevil

\begin{abstract}
Catolaccus grandis (Burks) is an important biological control agent of Anthonomus grandis (Boheman). The objective of this work was to evaluate biological aspects of that parasitoid using $A$. grandis larval as host, at $26\left(2^{\circ} \mathrm{C}, 70\right.$ ( $5 \% \mathrm{RH}$, and $12 \mathrm{~h}$ photophase. The total life cycle of the parasitoid was 16.2 days. Incubation period lasted 1.2 days with egg viability of $100 \%$. Larval stage showed three instars with a total duration of 7.2 days and viability of $97.2 \%$. Duration of pupal period stage was 8.0 days, and viability $96.3 \%$. The preovipositional period was 4.0 days; the oviposition period was 35.0 days; average number of eggs/female was 308.0; the average daily oviposition rate was $8.8 \mathrm{eggs} /$ female. Longevity of $C$. grandis was 51.0 days for females and 40.0 days for males.
\end{abstract}

KEY WORDS: Insecta, Anthonomus grandis, Gossypium hirsutum L., biological control.

RESUMO - Estudou-se em laboratório, aspectos biológicos do parasitóide Catolaccus grandis (Burks) utilizando como hospedeiro larvas de Anthonomus grandis (Boh.). O estudo foi conduzido a $26\left(2^{\circ} \mathrm{C}, 70\right.$ ( $5 \%$ de UR e $12 \mathrm{~h}$ fotofase. O ciclo biológico total do parasitóide foi de 16,2 dias. O período de incubação foi de 1,2 dias, com viabilidade dos ovos de $100 \%$. A fase larval apresentou três instares com duração total de 7,2 dias e viabilidade de 97,2\%. A duração pupal foi de 8,0 dias e a viabilidade foi de $96,3 \%$. O período de pré-oviposição de 4,0 dias; o período de oviposição 35,0 dias o número de ovos colocados por fêmea 308,0; a oviposição média diária foi 8,8 ovos/fêmea/dia. A longevidade de $C$. grandis foi 51,0 dias para fêmea e 40,0 dias para machos.

PALAVRAS-CHAVE: Insecta, Anthonomus grandis, Gossypium hirsutum L., controle biológico.

O bicudo do algodoeiro, Anthonomus grandis (Boheman) tem agentes biológicos de controle natural importantes. Entre estas espécies encontra-se uma série de parasitóides de larvas do bicudo (Chesnut \& Cross, 1971) entre os quais se destaca o ectoparasitóide Catolaccus grandis (Burks), descrito a partir de exemplares coletados na Nicarágua, o qual 
tem despertado o interesse dos entomologistas, por sua alta especificidade sobre $A$. grandis (Burks 1954) e suas características intrínsecas, que fazem com que seja um agente promissor de controle biológico para se tentar sua criação massal e, posteriormente, liberação no campo (Johnson et al. 1973). Este autor menciona que C. grandis tem potencial reprodutivo comparável ao de $A$. grandis. Este parasitóide tem sido reportado parasitando o bicudo na Costa Rica, Nicarágua, Guatemala e México (Cross \& Mitchell 1969). Em alguns países tem-se feito introdução deste parasitóide, principalmente nos Estados Unidos (Cate 1985, Cate et al. 1990) e, embora os mesmos tenham sido eficientes, não se estabeleceram, devido às baixas temperaturas.

No Brasil, tem havido maior interesse dos pesquisadores no estudo de parasitóides do bicudo, principalmente devido os recentes registros desses agentes biológicos reguladores das populações da praga ocorrendo naturalmente na cultura de algodão Gossypium hirsutum (Bleicher \& BroglioMicheletti 1988, Ramalho \& Silva 1993, Ramalho \& Wanderley 1995). A larva de $C$. grandis desenvolve externamente no $3^{\circ}$ ínstar do bicudo (Johnson et al. 1973). As fêmeas depositam de um a cinco ovos nas laterais ou sobre as larvas de bicudo e, pode parasitar 366,0 larvas do hospedeiro durante sua vida (Morales-Ramos 1991). No Brasil, são escassos os estudos sobre este parasitóide; assim, o trabalho foi desenvolvido com o objetivo de se estudar os aspectos biológicos de C. grandis visando à obtenção de subsídios para a sua criação massal.

\section{Material e Métodos}

O estudo foi desenvolvido no Laboratório de Entomologia da Embrapa Algodão, em Campina Grande, PB, em câmara climatizada regulada a $26\left(2^{\circ} \mathrm{C}\right.$, UR de 70 ( $5 \%$ e fotofase de 12 horas. Para obtenção dos ovos, foram separadas 118 fêmeas recém-emergidas de $C$. grandis de uma criação mantida no laboratório, os quais foram individualizadas em tubos de vidro $(8,5 \times 2,5 \mathrm{~cm})$ tampados com algodão e alimentados com uma solução de mel a 20\%; nesses tubos foram colocadas individualmente, oito larvas de 30 ínstar de A. grandis em cavidades feitas nas película de parafilm ( como descrito por Cate (1987) e oferecidas às fêmeas do parasitóide para oviposição. Após seis horas de confinamento, separaram-se as larvas parasitadas de A. grandis em novas cavidades da película, deixando-se, sobre as mesmas, um ovo do parasitóide, totalizando 114 ovos. Os tubos contendo estas películas foram mantidos em câmaras até emergência dos adultos.

O número de ínstares larvais de $C$. grandis foi determinado marcando-se a cutícula da larva do parasitóide com um corante de contato (Day-Glowâ). A mudança de ínstar foi confirmada com a ausência do marcador na cutícula e a presença da exúvia do parasitóide marcada. De acordo com a data de emergência dos adultos de $C$. grandis, estes foram sexados e acasalados para determinação dos períodos de pré-oviposição, oviposição, pós-oviposição, número de ovos/fêmea, número de ovos/fêmea/dia e longevidade. Realizou-se também a determinação da razão e proporção sexual, de acordo com Silveira Neto et al. (1976).

\section{Resultados e Discussão}

Sobrevivência e Desenvolvimento. O período de incubação foi de 1,2 dias (Tabela $1)$, com viabilidade de $100 \%$, sendo encontrado valor semelhante por Johnson et al. (1973) para $C$. grandis, de 27 a 29 horas, a $24-27^{\circ} \mathrm{C}$. O período médio larval completouse em 7,2 dias, com viabilidade de 97,2\%, apresentando três ínstares, com períodos de duração de 2,3 dias com viabilidade de 97,5\%; 1,5 dias, com viabilidade de 93,9\% e 3,3 dias com viabilidade de $97,2 \%$ para o primeiro, segundo e terceiro ínstares, respectivamente (Tabela 1). Johnson et al. (1973) observaram para a referida espécie uma fase larval um pouco mais curta (4,5 a 5,5 dias).

Comparando-se este resultado com os obtidos por Morales-Ramos (1991) verifica- 
Tabela 1. Duração $(\mathrm{X} \pm \mathrm{EP})$ e viabilidade de cada fase de desenvolvimento de fêmeas de Catalocus grandis criada com Anthonomus grandis.

\begin{tabular}{lcc}
\hline Fases & Duração (dias) & Viabilidade (\%) \\
\hline Incubação (ovos) & $1,2 \pm 0,0$ & 100,0 \\
$1^{\circ}$ ínstar & $2,3 \pm 0,0$ & 97,5 \\
$2^{\circ}$ ínstar & $1,5 \pm 0,0$ & 93,9 \\
$3^{\circ}$ ínstar & $3,3 \pm 0,0$ & 97,2 \\
$1^{\circ}$ a 30 ínstares & $7,2 \pm 0,0$ & 97,2 \\
Pupa & $8,0 \pm 0,1$ & 96,3 \\
Ovo-adulto & $16,2 \pm 0,2$ & 96,3 \\
\hline
\end{tabular}

se que a duração média da fase larval foi de 158,0 horas compreendendo cinco ínstares à $30^{\circ} \mathrm{C}$.

Durante o período pupal observou-se que a pupa recém-formada tem coloração brancocremosa, depois vai tornando marrom-claro, até ficar totalmente preta, iniciando-se pelo tórax e, posteriormente, pelo abdômen; a duração média foi de 8,0 dias (Tabela 1) e viabilidade de $96,3 \%$. Apresenta dimorfismo sexual, podendo, neste estágio, ser feita a sexagem. O período do ciclo total (ovoadulto) obtido neste trabalho foi de 16,2 dias para fêmeas, com viabilidade de $96,3 \%$ (Tabela 1).

Períodos Reprodutivos. O período de préoviposição durou, em média, 4,0 dias, e variou

Tabela 2. Duração $(X \pm E P)$ das diferentes fases do estágio adulto de Catalocus grandis, criado com Anthonomus grandis.

\begin{tabular}{lc}
\hline Estágios & Duração (dias) \\
\hline Pré-oviposição & $4,0 \pm 0,1$ \\
Oviposição & $35,0 \pm 0,3$ \\
Pós-oviposição & $4,5 \pm 0,1$ \\
$\mathrm{~N}^{\circ}$ ovos/fêmea & $308,0 \pm 3,5$ \\
$\mathrm{~N}^{\circ}$ ovos/fêmea/dia & $8,8 \pm 0,2$ \\
Longevidade (fêmea) & $51,0 \pm 0,3$ \\
\hline
\end{tabular}

de 1 a 12 dias. A duração do período de oviposição foi 35,0 dias, variando de 5 a 65 dias (Tabela 2), estes resultados foram inferiores ao verificado por Morales-Ramos (1991). A duração média de pós-oviposição foi de 4,5 dias, variando de 1 a 16 dias. A longevidade média de fêmeas de $C$. grandis foi de 51,0 dias, variando de 18 a 85 dias (Tabela 2).

Fecundidade e Razão Sexual. Pelos dados que constam na Tabela 2, observa-se que o número de ovos/fêmea foi em média 308,0 variando de 8-941, assemelhando-se também aos dados de Johnson et al. (1973) que obtiveram fecundidade média de 315 ,0 ovos/ fêmea. Com relação a oviposição média diária obteve-se 8,8 ovos/fêmea/dia. Cole \& Adkisson (1981) observaram que as fêmeas de A. grandis ovipositaram 6,5 ovos/dia a $26,7^{\circ} \mathrm{C}$. Comparando o valor médio deste experimento ( 8,8 ovos/fêmea/dia), verifica-se que $C$. grandis, aparentemente produz um número de ovos por dia superior ao seu hospedeiro, nas condições em que foi feito o experimento. Assim, considera-se que $C$. grandis, provavelmente será um potencial controlador desta praga. A proporção sexual (fêmea:macho) de C. grandis foi de 1:0,96. 


\section{Literatura Citada}

Bleicher, E. \& S.M. Broglio-Micheletti. 1988. Parasitos e predadores do bicudodo-algodoeiro no Nordeste do Brasil. p.130. In Relatório Técnico Anual da EMBRAPA - Centro Nacional de Pesquisa do Algodão - 1985/1986, Campina Grande, 185p.

Burks, B.D. 1954. Parasitic wasps of the Catolaccus grandis in the Americas. USDA Tech. Bull. 1093: 1 - 21.

Cate, J.R. 1987. A method of rearing parasitoides of boll weevil without the host plant. Southwest. Entomol. 12:211215.

Cate, J.R. 1985. Cotton: Status and current limitations to biological control in Texas and Arkansas, pp. 537-556. In M.A. Hoy \& D.C. Herzog (eds.), Biological control in agricultural IPM systems. Academic, New York.

Cate, J.R., P.C. Krauter \& K.E. Godfrey. 1990. Pests of cotton, pp. 17-29. In D.H. Habeck, F.D. Bennett \& J.H. Frank (eds.), Classical biological control in the southern United States. South. Coop. Ser. Bull. 355.

Chesnut, T.L. \& W.H. Cross. 1971. Arthropod parasites of boll weevil, Anthonomus grandis: 2. Comparisons of their importance in the United States over a period of thirty - eight years. Ann. Entomol. Soc. Am. 64:549-557.

Cole, C.L. \& P.L. Adkisson. 1981. Life history and fecundity of the boll weevil reared in constant and variable temperature regimens. Southwest. Entomol. 6:298-303.

Cross, W.H. \& H.C. Mitchell. 1969. Distribution and importance of Heterolaccus grandis as a parasite of the boll weevil. Ann. Entomol. Soc. Am. 62: 235-236.

Johnson, W.L., W.H. Cross., W.L. McGovern. \& H.C. Mitchell. 1973. Biology of Heterolaccus grandis in a laboratory culture and its potential as an introduced parasit of the boll weevil in the United States. Environ Entomol. 2:112-118.

Morales-Ramos, J.A. 1991. Rate of increase, functional response, and development of Catolaccus grandis (Burks) (Hymenoptera: Pteromalidae). Tese de doutorado, Texas A \& M University, Austin, 126p.

Ramalho, F.S. \& J.R.B. Silva. 1993. Período de emergência e mortalidade natural do bicudo-do-algodoeiro. Pesq. Agropec. Bras. 28: 1221-1231.

Ramalho, F.S. \& P.A. Wanderley. 1995. Ecology and management of the boll weevil. In South American Cotton. Am. Entomol. 42: 41-47.

Silveira Neto, S., O. Nakano, D. Barbin \& N.A. Vila Nova. 1976. Manual de ecologia dos insetos. Piracicaba, Ed. Agronomica Ceres, 419p.

Recebido em 24/09/96. Aceito em 06/10/97. 